Adnan H. Siddiqui, MD, $\mathrm{PhD}$

Robert Zivadinov, MD, $\mathrm{PhD}$

Ralph H.B. Benedict, $\mathrm{PhD}$

Yuval Karmon, MD

Jihnhee $\mathrm{Yu}, \mathrm{PhD}$

Mary L. Hartney, RN, CCRC

Karen L. Marr, RVT, RDMS

Vesela Valnarov, MD, RVT, ARDMS, RPVI Cheryl L. Kennedy, LMSW, MPH

Murali Ramanathan, PhD Deepa P. Ramasamy, MD Kresimir Dolic, MD

David W. Hojnacki, MD Ellen Carl, MA

Elad I. Levy, MD, MBA

L. Nelson Hopkins, MD Bianca Weinstock-

Guttman, MD

Correspondence to Dr. Siddiqui: asiddiqui@ubns.com or Dr. Zivadinov: rzivadinov@bnac.net

Editorial, page 388

Supplemental data at Neurology.org

\title{
Prospective randomized trial of venous angioplasty in MS (PREMiSe)
}

金

\section{ABSTRACT}

Objective: We report the results of the investigation of safety and efficacy of venous angioplasty in patients with multiple sclerosis (MS) with findings of extracranial venous anomalies, considered hallmarks of chronic cerebrospinal venous insufficiency (CCSVI), in a 2-phase study (ClinicalTrials.gov NCT01450072).

Methods: Phase 1 was an open-label safety study (10 patients); phase 2 was sham-controlled, randomized, and double-blind (10 sham procedure, 9 treated). All study patients fulfilled venous hemodynamic screening criteria indicative of CCSVI. Assessment was at 1, 3, and 6 months postprocedure with MRI, clinical, and hemodynamic outcomes. Primary endpoints were safety at 24 hours and 1 month, venous outflow restoration $>75 \%$ at 1 month, and effect of angioplasty on new lesion activity and relapse rate over 6 months. Secondary endpoints included changes in disability, brain volume, cognitive tests, and quality of life.

Results: No perioperative complications were noted; however, one patient with history of syncope was diagnosed with episodic bradycardia requiring placement of a pacemaker before discharge. Doppler evidence-based venous hemodynamic insufficiency severity score (VHISS) was reduced $>75 \%$ compared to baseline in phase 1 (at 1 month) but not phase 2. In phase 2, higher MRI activity (cumulative number of new contrast-enhancing lesions [19 vs 3, $p=0.062$ ] and new T2 lesions [17 vs $3, p=0.066]$ ) and relapse activity ( 4 vs $1, p=0.389$ ) were identified as nonsignificant trends in the treated vs sham arm over 6 months. Using analysis of covariance, significant cumulative new T2 lesions were related to larger VHISS decrease $(p=0.028)$ and angioplasty ( $p=0.01$ ) over the follow-up. No differences in other endpoints were detected.

Conclusion: Venous angioplasty is not an effective treatment for MS over the short term and may exacerbate underlying disease activity.

Classification of evidence: This is a Class I study demonstrating that clinical and imaging outcomes are no better or worse in patients with MS identified with venous outflow restriction who receive venous angioplasty compared to sham controls who do not receive angioplasty. This study also includes a Class IV phase 1 study of safety in 10 patients receiving the angioplasty procedure. Neurology ${ }^{\circledR}$ 2014;83:441-449

\section{GLOSSARY}

$\mathbf{A E}=$ adverse event; $\mathbf{A N C O V A}=$ analysis of covariance; $\mathbf{A N O V A}=$ analysis of variance; $\mathbf{C C S V I}=$ chronic cerebrospinal venous insufficiency; $\mathbf{C E}=$ contrast-enhancing; $\mathbf{C V}=$ catheter venography; EDSS = Expanded Disability Status Scale; IJV = internal jugular vein; IVUS = intravascular ultrasound; MS = multiple sclerosis; MSFC = MS Functional Composite; PREMiSe = Prospective Randomized Endovascular Therapy in MS; QoL = quality of life; VH = venous hemodynamic; VHISS $=$ venous hemodynamic insufficiency severity score.

Multiple sclerosis (MS) is characterized by demyelinating lesions affecting the CNS. An association between MS and extracranial venous outflow restrictive lesions detected by venous duplex studies, named chronic cerebrospinal venous insufficiency (CCSVI), has been described. ${ }^{1}$ There is controversy as to the nature of these structural and functional extracranial venous anomalies and whether they even represent pathologic findings, and certainly no agreement exists as to whether there is any etiologic relationship with MS. Several recent prevalence studies that used

From the Departments of Neurosurgery and Radiology, The Jacobs Neurological Institute (A.H.S., Y.K., M.L.H., E.I.L., L.N.H.), and the Departments of Neurology (R.Z., R.H.B.B., D.W.H., B.W.-G.), Biostatistics (J.Y.), and Pharmaceutical Sciences (M.R.), University at Buffalo, State University of New York; and the Buffalo Neuroimaging Analysis Center (R.Z., K.L.M., V.V., C.L.K., D.P.R., K.D., E.C.), NY.

Go to Neurology.org for full disclosures. Funding information and disclosures deemed relevant by the authors, if any, are provided at the end of the article. 
different imaging techniques have reported large discrepancies in the prevalence of extracranial venous anomalies characterized as CCSVI findings in patients with MS. ${ }^{2-7}$

Endovascular treatment for these venous anomalies was introduced in an open-label study that included 65 patients with MS with postprocedure follow-up of $>18$ months. ${ }^{8}$ Several subsequent prospective open-label, nonrandomized studies investigated safety and efficacy of venous angioplasty in MS. ${ }^{9-18}$ Findings from some of these studies have generated considerable controversy over potential treatment benefit, which remains unproven, whereas others showed a potential increase in disease activity. ${ }^{9,10,19}$

We investigated safety and efficacy of venous angioplasty in patients with MS exhibiting findings of extracranial venous outflow restrictive anomalies described as hallmarks of CCSVI in the setting of a prospective, double-blind, sham-controlled, randomized pilot trial. Primary study endpoints were safety, venous outflow restoration, and effect of angioplasty on MRI lesion activity and relapse rate.

METHODS Study design and patient selection. Prospective Randomized Endovascular Therapy in MS (PREMiSe) (ClinicalTrials.gov NCT01450072) was planned in 2 phases. Phase 1 was an open-label safety study of endovascular venous angioplasty with an intended enrollment of 10 patients with MS with extracranial venous anomalies consistent with criteria utilized for describing CCSVI and was conducted to strengthen procedural protocols and work out blinding methodologies. Phase 2 was sham-controlled, randomized, and double-blind, including up to 20 patients with MS with CCSVI type venous anomalies undergoing either angioplasty or sham procedure. The sample size was restricted owing to the pilot nature of this study because there were no previous studies evaluating effects of angioplasty in patients with MS with control subjects. We assumed a 50\% treatment effect to keep a small sample size in view of the pilot nature of the purported effects published at the time this study was designed. Both phases were of 6 months' duration. Patients were enrolled between June 2010 and March 2012.

Standard protocol approval, registrations, and patient consents. The study was approved by our Institutional Review Board and overseen by a data safety monitoring committee consisting of physicians not involved in the care or treatment of patients with MS at the University at Buffalo. Written informed consent was obtained from all subjects. Screening, diagnostic, interventional, and follow-up procedures and visits were performed at no cost to the patients. Data were collected by the investigators and analyzed by an independent statistician.

Inclusion criteria for phase 1 were as follows: age 18-65 years, Expanded Disability Status Scale (EDSS) score ${ }^{20} 0-8.5$, diagnosis of clinically definite MS, ${ }^{21}$ and fulfilling at the time of screening $\geq 2$ CCSVI venous hemodynamic $(\mathrm{VH})$ duplex criteria. ${ }^{22}$ Inclusion criteria for phase 2 were refined further to identify patients most demonstrative of structural and hemodynamic dysfunction described as CCSVI and most likely to benefit from intervention: age $18-65$ years, EDSS score ${ }^{20} 0-5.5$, activerelapsing $\mathrm{MS},{ }^{23}$ and fulfilling, at the time of screening, $\geq 2$ VH extracranial duplex criteria. ${ }^{22}$ Details of venous duplex screening are provided in appendix e-1 on the Neurology ${ }^{\circledR}$ Web site at Neurology.org. Active-relapsing disease was defined as 1 relapse within the past 12 months or presence of contrastenhancing (CE) lesions on postcontrast MRI within the previous 3 months and concomitant treatment with disease-modifying treatments excluding natalizumab.

Exclusion criteria (either phase) included acute relapse, disease progression, or steroid treatment within 30 days preceding study entry, preexisting medical conditions known to be associated with brain pathology (e.g., neurodegenerative disorder, cerebrovascular disease, history of alcohol abuse), severe peripheral chronic venous insufficiency, severe contrast media allergy (anaphylaxis), and abnormal renal function.

Patients were also required to fulfill screening criteria on catheter venography $(\mathrm{CV})$, defined as azygous vein or internal jugular vein (IJV) luminal diameter reduction $\geq 50 \%$. CV findings were confirmed by intravascular ultrasound (IVUS); both studies were performed under conscious sedation with local anesthesia preceding the endovascular venous angioplasty or sham procedure. $\mathrm{CV}$ was conducted using the method previously described ${ }^{8}$ and interpreted by interventional neurosurgeons (A.H.S., E.I.L., L.N.H.). Diagnostic CV is described briefly in appendix e-2 and in detail elsewhere. $^{24}$

In phase 2, randomization was performed by an independent statistician in 1:1 fashion and is described in appendix e-3. All study personnel, with the exception of the interventional neurosurgeons, were blind to the assigned procedure, as were the patients. Blinding is described in appendix e-3.

Sham and venous angioplasty. The goal of angioplasty was to restore venous outflow of stenotic IJVs and azygous vein to $>50 \%$ of normal proximal venous diameter at the time of intervention. Angioplasty was performed only in the treated, not in the sham, arm. To ensure proper blinding, all patients received a rigorous sternal rub (painful stimulus) upon insertion of the angioplasty balloon, but the balloon was inflated only in the treated arm; all were prevented from observing fluoroscopic images. A detailed description of these procedures is provided in appendix e- 4 .

Endpoints and follow-up assessment. The primary endpoint was safety at 24 hours and 1 month. A serious adverse event (AE) was defined as an untoward medical occurrence that was life-threatening, resulted in persistent disability or required intervention to prevent it, caused prolongation of hospitalization, or resulted in death. Nonserious AEs were all others. Preliminary efficacy outcomes were venous outflow restoration of $>75 \%$ at 1 month compared to baseline, as measured by changes in venous hemodynamic insufficiency severity score (VHISS), and effect of angioplasty on new MRI-based lesion activity and clinical relapse rate over 6 months. Additional endpoints included changes in EDSS, brain volume, cognitive tests, 6-minute walk, and quality of life (QoL), as well as MS Functional Composite (MSFC) scores. A detailed description of study endpoints is provided in appendix e-5.

Statistical analysis. Statistical analysis was performed using the Statistical Package for the Social Sciences (version 17.0; SPSS, Chicago, IL) and Statistical Analysis System (Version 9.3; SAS, Cary, NC). The normal assumption was inspected using the normal quantile plot and histogram based on the residuals from respective models used. To handle digresses from the normal distribution, alternative methods based on nonparametric methodologies (e.g., Mann-Whitney $U$ test and a previously described 
Table 1 Baseline demographic, clinical, and duplex characteristics of patients enrolled in the PREMiSe study

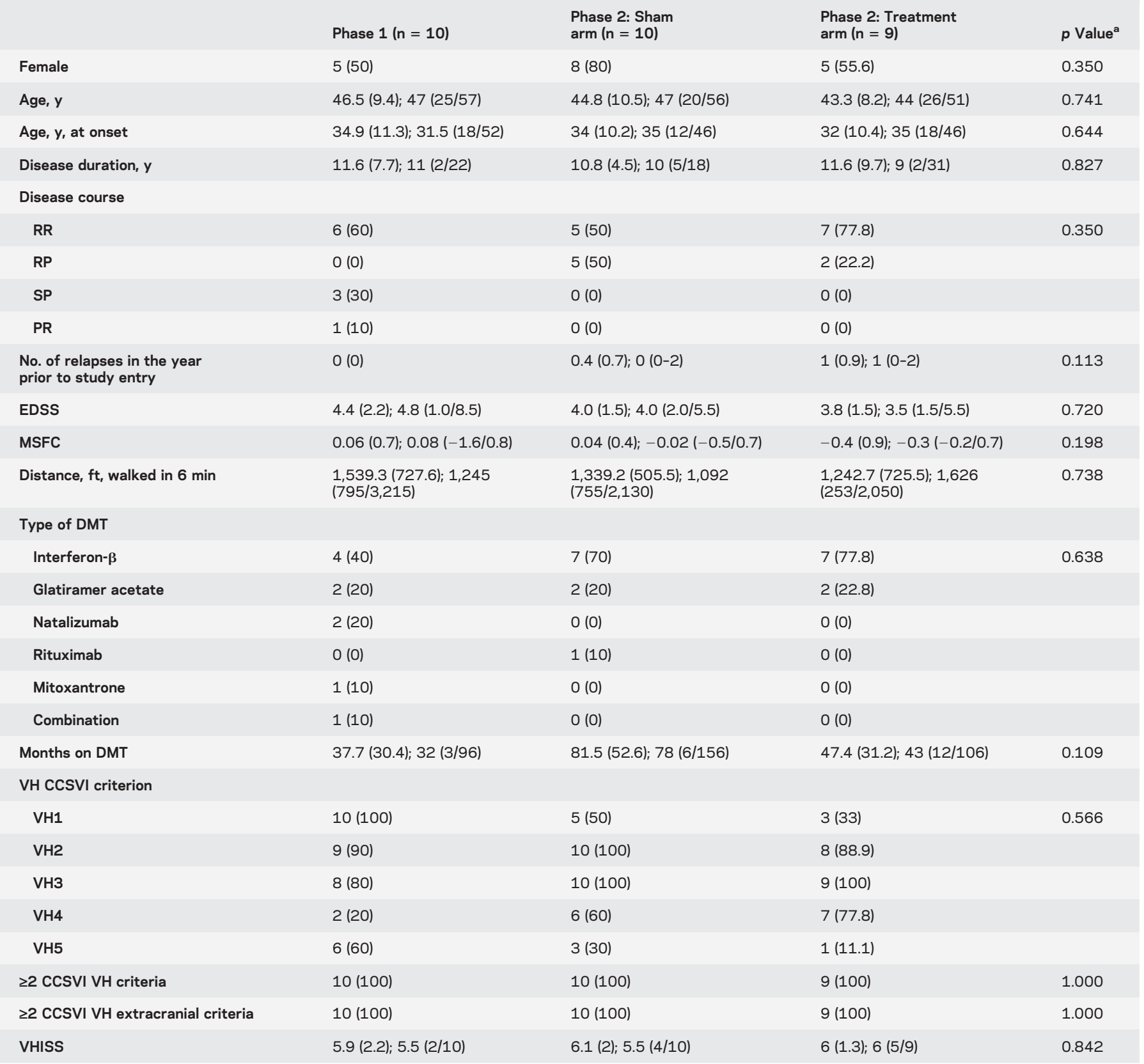

Abbreviations: CCSVI = chronic cerebrospinal venous insufficiency; DMT = disease-modifying therapy; EDSS = Expanded Disability Status Scale; MSFC = Multiple Sclerosis Functional Composite; PREMiSe = Prospective Randomized Endovascular Therapy in MS; PR = progressive relapsing; RP = relapsingprogressive; RR = relapsing-remitting; $\mathrm{SP}=$ secondary progressive; $\mathrm{VH}=$ venous hemodynamic; VHISS = venous hemodynamic insufficiency severity score.

Values are mean (SD); median (min/max) or $\mathrm{n}(\%)$.

${ }^{a} p$ Value represents statistical analysis between sham and treated arms of phase 2. Analysis between these groups was performed by using $\chi^{2}$ test, Student $t$ test, and Mann-Whitney rank sum test, as appropriate.

method $^{25}$ ) were used. Statistical analyses included comparisons between only the 2 treatment arms in phase 2. For demographic, clinical, and MRI differences between the 2 groups, Student $t$ tests, $\chi^{2}$ tests, and Mann-Whitney $U$ tests were used as appropriate.

Multi-timepoint longitudinal changes over 6 months in clinical, MRI, QoL, and cognitive outcomes were analyzed by using mixed-effects analysis of variance (ANOVA) models that include different treatment groups, month (as a categorical variable), and their interaction as factors. Mixed-effects ANOVA included baseline outcomes as dependent variables so that a significant interaction between month and treatment arms would indicate treatment differences. With no presence of the interaction, the $p$ value for month in the mixed-effects ANOVA was used for overall time effect (based on all phase 2 patients). Means and confidence intervals in figure plots are based on raw data. To test whether relapse rate and MRI lesion activity outcomes were dependent on venous outflow restoration success in phase 2, we applied logistic regression analysis or analysis of covariance (ANCOVA), in which VHISS and treatment status were used as covariates. Given the exploratory pilot nature of the study, a nominal $p$ value of $<0.05$ was considered significant using 2-tailed tests. 
Table 2 Adverse events in patients enrolled in PREMiSe over 6 months

\begin{tabular}{|c|c|c|c|}
\hline & Phase $1(n=10)$ & Phase 2: Sham arm $(n=10)$ & Phase 2: Treatment arm $(n=9)$ \\
\hline \multirow[t]{5}{*}{ Description of $A E$} & $\begin{array}{l}\text { 1. Rash due to Doppler } \\
\text { sonography at screening }\end{array}$ & $\begin{array}{l}\text { 1. Immune thrombocytopenic purpura } \\
\text { treated with } 100 \mathrm{mg} \text { prednisone once daily }\end{array}$ & $\begin{array}{l}\text { 1. Cardiac event treated with } \\
\text { pacemaker installation }\end{array}$ \\
\hline & $\begin{array}{l}\text { 2. UTI treated with } \\
\text { antibiotics for } 10 \text { days }\end{array}$ & $\begin{array}{l}\text { 2. Bladder infection treated with } \\
\text { antibiotics over } 10 \text { days }\end{array}$ & $\begin{array}{l}\text { 2. Swelling and soreness at left } \\
\text { side of the neck; no treatment required }\end{array}$ \\
\hline & $\begin{array}{l}\text { 3. UTI treated with } \\
\text { antibiotics for } 5 \text { days }\end{array}$ & $\begin{array}{l}\text { 3. Diagnosis of shingles treated } \\
\text { with Valtrex (GlaxoSmithKline) three } \\
\text { times daily for } 7 \text { days }\end{array}$ & $\begin{array}{l}\text { 3. Hospitalization for scheduled } \\
\text { transobturator sling procedure }\end{array}$ \\
\hline & $\begin{array}{l}\text { 4. Intercourse pain } \\
\text { (condom-related) that } \\
\text { prompted hospitalization } \\
\text { for } 2 \text { days }\end{array}$ & & \\
\hline & $\begin{array}{l}\text { 5. Neck pain due to } \\
\text { car accident }\end{array}$ & & \\
\hline \multirow[t]{5}{*}{ Severity of $A E$} & 1. Nonserious & 1. Serious & 1. Serious \\
\hline & 2. Non & 2. Non & 2. Non \\
\hline & 3. Non & 3. Non & 3. Non \\
\hline & 4. Non & & \\
\hline & 5. Non & & \\
\hline \multirow[t]{5}{*}{ Timepoint of AE } & 1. Baseline & 1. 6 months & 1. 24 hours \\
\hline & 2. 6 months & 2. 6 months & 2. 6 months \\
\hline & 3. 6 months & 3. 6 months & 3. 6 months \\
\hline & 4. 6 months & & \\
\hline & 5. 6 months & & \\
\hline \multirow{5}{*}{$\begin{array}{l}\text { Relationship of } A E \text { to treatment } \\
\text { or invasive diagnostic procedure }\end{array}$} & 1. Unrelated & 1. Unrelated & 1. Unrelated \\
\hline & 2. Unrelated & 2. Unrelated & 2. related \\
\hline & 3. Unrelated & 3. Unrelated & 3. Unrelated \\
\hline & 4. Unrelated & & \\
\hline & 5. Unrelated & & \\
\hline
\end{tabular}

Abbreviations: AE = adverse event; PREMiSe = Prospective Randomized Endovascular Therapy in MS; UTI = urinary tract infection. AEs are listed in chronological order with individual AEs assigned an increasing number.

RESULTS Screening, randomization, and blinding. In total, 15 patients signed informed consent in phase 1 and 30 in phase 2 after prescreening qualification procedures were completed. Of those, 5 in phase 1 and 10 in phase 2 did not fulfill noninvasive screening procedure requirements on duplex examination. As preplanned, 10 patients were enrolled in phase 1 and 20 in phase 2 . Of those, 1 patient in phase 2 did not fulfill invasive screening criteria for endovascular intervention. Hence, 10 patients were randomized to the sham treatment arm and 9 to the angioplasty treatment arm and received the allocated intervention in phase 2 (figure e-1).

Baseline characteristics. The phase 2 treatment arms were reasonably well-matched for various demographic, clinical, and duplex characteristics with no statistically significant between-group differences (table 1). Phase 1 patients were also well-matched to phase 2 patients, except for inclusion of 3 secondaryprogressive patients and lower number of relapses in the previous year. All groups were also well-matched with respect to MRI and clinical metrics (table e- 1 and table e-2).

Safety and tolerability of treatment procedures. All patients in phases 1 and 2 tolerated the endovascular procedure well. No operative or postoperative complications (vessel rupture, thrombosis, or side effects to contrast media) were identified. No serious AEs were detected at any timepoint in phase 1 (table 2). Half the phase 1 patients reported a nonserious AE; none was related to the treatment procedure (table 2).

In phase 2,1 patient in the treated arm presented with a serious $\mathrm{AE}$ at 24 hours (table 2). The patient experienced an episode of symptomatic bradycardia that was confirmed by telemetry; consequently, a cardiac consultation recommended pacemaker installation. During pretreatment screening, bradycardia was not noted on electrocardiography or by history. However, before pacemaker installation, the patient confirmed previous similar episodes not reported to any physicians. Further follow-up was uneventful for this patient. Although considered preexistent, this event 
Figure 1 Graphic representation of venous outflow dilation outcomes

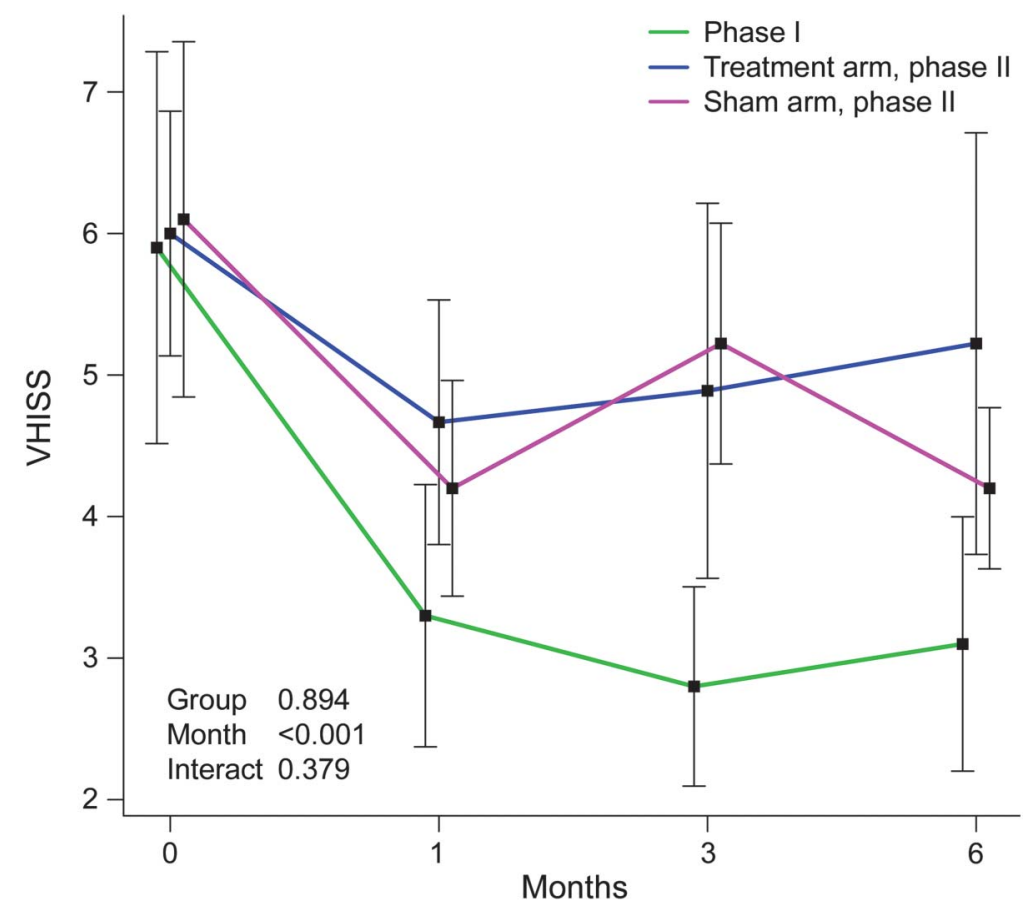

Changes in venous hemodynamic insufficiency severity score (VHISS) at 1, 3, and 6 months, compared to baseline, in phases 1 and 2 plotted by using mixed-effect model analysis. $p$ Values in the plot are based on comparison between phase 2 groups. Time effect $p$ values within groups are phase $1(p<0.0001)$, phase 2 treated arm $(p=0.02)$, and phase 2 sham arm $(p=0.04)$, respectively.

could be possibly related to venous angioplasty. Another patient, in the sham arm, presented with a serious $\mathrm{AE}$ at 6 months. The event was a viral infection causing immune thrombocytopenic purpura that was treated with prednisone $(100 \mathrm{mg} /$ day $)$ and was unrelated to the study.

Radiographic venous outflow dilation outcomes. We evaluated radiographic evidence of luminal enlargement following venous angioplasty. We noted that venous outflow could achieve at least $50 \%$ of proximal venous diameter in all phase 1 and 2 patients at the time of intervention as demonstrated by angiography. In phase 1 (figure 1), there was improvement of VHISS $(p<0.0001)$ over 6 months that resulted in $>75 \%$ restoration of venous outflow compared to baseline. In phase 2, improvement was observed also in treatment $(p=0.02)$ and sham $(p=0.04)$ arms at month 1 but did not reach $>75 \%$ restoration of venous outflow compared to baseline. No differences in VHISS improvement were detected between phase 2 treated and sham groups $(p=0.894)$.

Changes in clinical outcomes. No relapses occurred in phase 1 . In phase 2 , there were 4 relapses in the treated arm (among 3 patients) and 1 in the sham arm $(p=0.389)$. The relapses occurred at 1,3 (2 relapses), and 6 months in the treated arm and at 5 months in the sham arm. There was no statistical evidence that a higher number of relapses in the treated arm was related to VHISS changes $(p=0.183)$ or angioplasty vs sham treatment status ( $p=0.401$ ). The relapse risk for phase 2 patients is shown by Kaplan-Meier plot in figure e-2.

No significant changes in EDSS, MSFC, or 6-minute walked distance were detected in phase 1 patients (figure e-3). In phase 2, no significant within- or between-group changes in EDSS, MSFC, or 6-minute walked distance were detected, except improvement of MSFC in the sham treatment arm ( $p=0.04$, figure e-3).

No significant between-group changes in cognitive or QoL outcomes were detected in phase 2 patients (figure e-4). However, in both phases, there were within-group changes in Symbol Digit Modalities Test ( $p=0.009$ for phase 2 treated arm), Beck Depression Inventory Fast Screen $(p=0.01$ for phase 2 sham arm), Fatigue Severity Scale ( $p=0.03$ for phase 2 sham arm), MS Neuropsychological Screening Questionnaire ( $p=0.008$ for phase 2 sham arm), and Multiple Sclerosis Quality of Life-54 physical ( $p=$ 0.02 for phase $1, p=0.0008$ for phase 2 treated arm, and $p=0.001$ for phase 2 sham arm) and mental health $(p=0.003$ for phase 2 sham arm) composites (figure e-3).

Changes in MRI outcomes. Two patients in phase 1 had MRI findings indicative of disease activity (table 3). Of 9 patients in the phase 2 treated arm, 5 showed new CE lesions, with 2 accounting for most of the lesion activity, and 4 of those 5 patients had new T2 lesions (table 3), whereas only 2 in the sham arm showed new lesion activity. There was a trend for higher cumulative number of new CE lesions ( $p=$ $0.062)$ and new T2 lesions $(p=0.066)$ in the treated compared to the sham arm over 6 months (figure 2).

Using mixed-effects ANOVA models, no significant interactions between month (postprocedure) and group or group effects were found. In separate analyses based on cumulative number of new lesions using ANCOVA, there was evidence that higher cumulative number of new T2 lesions was related to larger decrease in VHISS $(p=0.028)$ and treated $\operatorname{arm}(p=0.01)$ over the follow-up. There was a higher accumulation of T2 lesion volume ( $p=0.04)$ in the treated compared to the sham arm (phase 2) over 6 months (table 3). No differences in brain volume changes over 6 months were found (table 3). The safety profile should be interpreted with caution given the pilot nature of this study.

DISCUSSION We performed venous angioplasty of extracranial venous outflow restrictions in patients with MS safely. This safety profile is diminished by the trial's pilot nature and small sample size. However, the procedural correction of venous outflow 
Table 3 Changes in MRI measures in the PREMiSe study over 6 months

\begin{tabular}{|c|c|c|c|c|}
\hline & Phase $1(n=10)$ & Phase 2: Sham arm $(n=10)$ & Phase 2: Treatment arm $(n=9)$ & $p$ Value $^{a}$ \\
\hline $\begin{array}{l}\text { Cumulative no. of new } \\
\text { T2 lesions, sum }\end{array}$ & $0.2(0.4) ; 0(0 / 1) 2$ & $0.3(0.7) ; 0$ (0/2) 3 & $2.1(2.9) ; 1$ (0/8) 17 & 0.066 \\
\hline T2-LV absolute change & $0.4(1) ; 0.01(-0.09 / 3.4)$ & $-0.2(0.4) ;-0.06(-0.8 / 0.6)$ & 0.5 (1.5); $0.06(-0.3 / 4)$ & NA \\
\hline T2-LV \% change & 1.3 (10.3); $0.6(-15.9 / 23.4)$ & $-4.7(11) ;-1.5(-21.4 / 12.2)$ & 13.9 (22.8); 2.9 (-10.6/45.8) & 0.04 \\
\hline $\begin{array}{l}\text { Cumulative no. of T1 } \\
\text { lesions, sum }\end{array}$ & 0 & $0.2(0.6) ; 0(0 / 2) 2$ & $0.8(0.9) ; 0.5$ (0/2) 6 & 0.144 \\
\hline T1-LV \% change & -2.9 (32.7); $2.5(-73 / 28)$ & $-14.6(33.6) ;-5.3(-100 / 14.1)$ & $-10.2(30.9)_{i}-8.3(-50 / 32.9)$ & 0.811 \\
\hline $\begin{array}{l}\text { Cumulative no. of CE } \\
\text { lesions, sum }\end{array}$ & $0.1(0.3) ; 0(0 / 1) 1$ & $0.3(0.7) ; 0(0 / 2) 3$ & 2.4 (3.2); 1 (0/9) 19 & 0.062 \\
\hline CE-LV absolute change & $-0.04(0.1) ; 0(-0.4 / 0)$ & $-0.04(0.1) ; 0(-0.4 / 0)$ & $-0.03(0.08) ; 0$ (-0.1/0.1) & NA \\
\hline CE-LV \% change & $-100(0) ;-100(-100 /-100)$ & $-94.1(8.3) ;-94.1(-100 /-88.3)$ & $34.4(186.3) ;-44(-100 / 247.1)$ & 0.262 \\
\hline PBVC & $-0.64(0.66) ;-0.65(-1.86 / 0.24)$ & $-0.74(0.93) ;-0.56(-2.5 / 0.51)$ & $-0.23(0.84) ;-0.45(-1.1 / 1.1)$ & 0.257 \\
\hline GMVC & $-2.1(1.2) ; 2(-4.4 /-0.1)$ & $-1.84(3.1) ;-2.3(-6.5 / 3.3)$ & $-0.53(1.6) ;-0.65(-2.6 / 1.99)$ & 0.320 \\
\hline WMVC & 0.9 (1.5); $0.51(-0.96 / 4.3)$ & $0.4(2.9) ; 1.2(-3.8 / 4.4)$ & $0.12(2.6) ; 0.22(-4 / 4)$ & 0.841 \\
\hline
\end{tabular}

Abbreviations: $\mathrm{CE}$ = contrast-enhancing; GMVC = gray matter volume change; LV = lesion volume; NA = not available; PBVC = percentage brain volume change; PREMiSe = Prospective Randomized Endovascular Therapy in MS; WMVC = white matter volume change.

Values are mean (SD); median $(\min / \max )$ or $\mathrm{n}(\%)$. Sum = total number.

${ }^{a} p$ Value represents statistical analysis between sham and treated arms of phase 2 . The analysis between these groups was performed by using Student $t$ test. Statistical analysis between T2-LV, T1-LV, and CE-LV was performed only for percentage changes. Changes between baseline and follow-up scans for whole brain, gray matter, and white matter volumes were calculated using the direct measurement technique; hence, no absolute, but only percentage volume changes are available. The absolute lesion volumes are presented in milliliters. Statistical analyses between phase 2 sham and treated arms were also adjusted for age, sex, disease duration, relapse rate in the year prior to study entry, and number of CE lesions at baseline. No significant differences were found between these arms, except for T2-LV \% change $(p=0.05)$. Of the 5 patients with active multiple sclerosis assigned to the treated arm in phase 2, cumulative numbers of CE lesions per patient over 6 months were as follows: 9, 5, 3, 1, and 1 (respectively). In the sham arm, one patient had 2 CE lesions and one had 1.

restriction appears transient and not associated with durable improvement in VHISS at 6 months. Further, there appears to be no significant improvement in clinical disease following angioplasty. Rather, there was a trend towards increasing disease activity in treated patients as measured by MRI outcomes and relapses. Our findings may be skewed by the limited sample size; however, numerous pilot phase $1 / 2 \mathrm{a}$ treatment trials in MS included similar sample size, $^{26-29}$ before the decision was made to proceed with phase $2 \mathrm{~b}$ trials on the basis of safety and preliminary efficacy findings.

Extracranial venous outflow anomalies describing the CCSVI theory have generated tremendous controversy, with sizeable peer-reviewed data arguing both merits and faults with the hypothesis. ${ }^{30,31}$ There has been a massive clinical rush towards employing venous angioplasty for the treatment of MS despite the lack of evidence for this intervention. Most opinions in favor of the treatment are based on sudden and robust improvement as self-reported by patients. ${ }^{8,11,12,17,18}$

We have previously reported on extracranial venous anomalies. ${ }^{24,32,33}$ Our studies, though suggestive of a venous role in many chronic neurologic diseases including MS, were not quite as strongly supportive as initially reported, ${ }^{1}$ nor quite as dismissive as results recently reported. ${ }^{34}$ However, given the initial remarkable results, ${ }^{8,11,12,16,17,35}$ we attempted to design a study that could address critical aspects of the venous outflow restriction playing a role in MS pathogenesis (CCSVI hypothesis). Therefore, we designed PREMiSe with a double-blinded, sham-controlled design — which was unique for CCSVI interventional studies-knowing that many aspects of this hypothesis remained unsubstantiated but with clearly established biases among physicians and patients.

Our phase 2 results suggest that these previous selfreported findings are largely related to placebo effects. Similarly, one potential explanation for improved VHISS in phase 1 as compared to phase 2 could be that duplex examiners knew this was an open-label phase in which all patients were treated. However, in phase 2, sham patients also slightly improved in VHISS (figure 1). Potential explanations for this finding include (1) modest reproducibility, even among highly trained technicians ${ }^{32,33}$; (2) functional and 


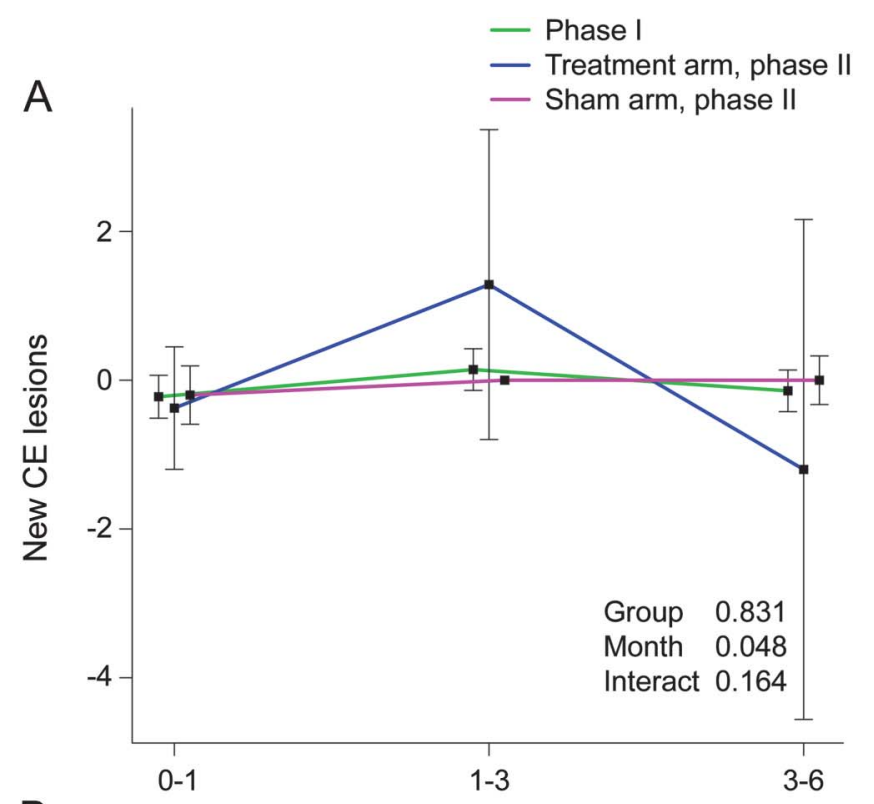

B
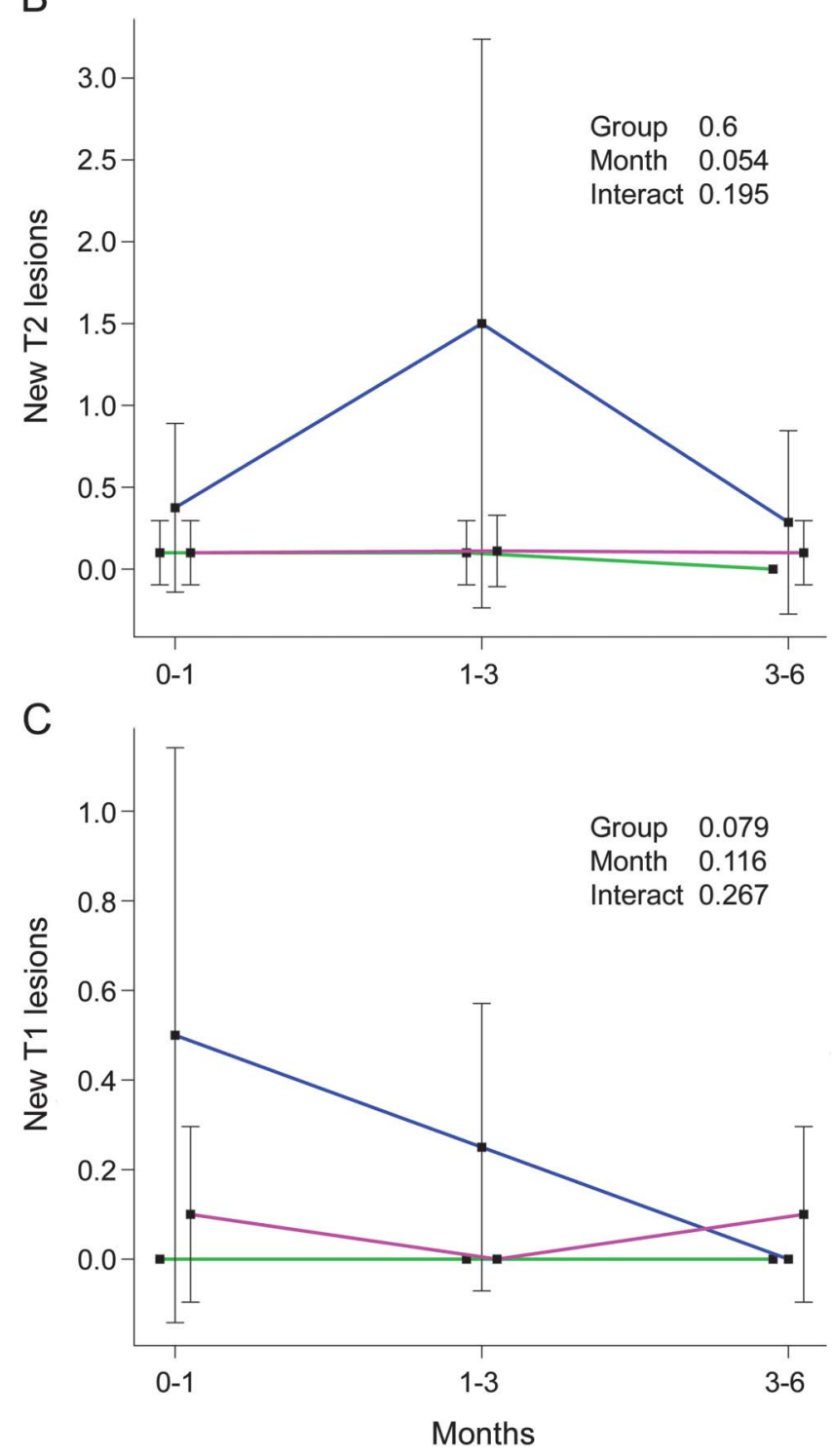

hemodynamic changes over time, possibly related to use of CV and IVUS during diagnostic procedures; and (3) administration of aspirin and enoxaparin sodium with ongoing pro/antithrombotic changes and potential effects on flow.

For the open-label component (phase 1), we were primarily focused on safety rather than efficacy. However, to target patients most likely to benefit from venous angioplasty, we restricted enrollment criteria for phase 2 by including patients who were less disabled (EDSS score 5 5.5) with active disease (relapse in the last 12 months or recent MRI activity) who demonstrated $\geq 2$ extracranial VH criteria. This rigor has not been applied in previous studies ${ }^{8,9,11-15,17,18}$ and despite our intention, it failed to demonstrate benefit of venous angioplasty.

Contrary to the proposed CCSVI hypothesis, analysis of VHISS changes suggests that a decrease in venous outflow restriction (i.e., improved venous outflow) correlated significantly with an increase in MRI activity. Due to the small sample size, only a trend in increase in clinical disease activity was noted with a reduction in VHISS scores. We have previously reported that lesion activity was increased after endovascular treatment for CCSVI in patients with MS. ${ }^{18}$ In line with these early observations, PREMiSe showed that of the 5 patients with MS in the treated arm (phase 2) with active MRI scans, 2 patients accounted for most lesion activity during the trial (table 3). Four possible hypotheses for explaining this paradoxical effect are as follows: (1) the patients did not respond to angioplasty; (2) reopening of the veins simply increases perfusion of the microcirculation of the brain parenchyma, which resulted in short-term increase in inflammation; (3) the observed azygous vein or IJV stenosis is a secondary compensatory response, such as in response to chronic venous reflux, that is exacerbated following venous angioplasty and results in increased disease activity; or (4) variability secondary to chance. In line with our findings, 2 recent retrospective studies show an increase in disease activity irrespective of adherence to disease-modifying

Changes in accumulation of mean new contrast-enhancing (CE) lesions (A), mean new T2 lesions (B), and mean T1 lesions $(C)$, between $0-1,1-3$, and 3-6 months in phases 1 and 2 , by using mixed-effect model analysis. Some means for new T2 and T1 lesions have standard error $=0$ since only values of 0 are presented. $p$ Values in the plot are based on comparison between the phase 2 groups. Time effect $p$ values within these groups are for mean new CE lesions: phase $1(p=0.198)$, phase 2 treated $\operatorname{arm}(p=$ $0.593)$, and phase 2 sham arm $(p=0.766)$, respectively; for mean new T2 lesions: phase $1(p=0.617)$, phase 2 treated $\operatorname{arm}(p=0.254)$, and phase 2 sham arm $(p=$ 0.991), respectively; and for mean new $T 1$ lesions: phase 2 treated arm $(p=0.057)$ and phase 2 sham $\operatorname{arm}(p=$ $0.776)$, respectively. 
therapy in patients with MS presenting with CCSVI who underwent venous angioplasty. ${ }^{9,10}$

No differences in cognitive or QoL outcomes were observed between the 2 treatment arms in shamcontrolled phase 2 . However, various outcome measures showed within-group improvements over 6 months in both phases and need further analysis.

In this first double-blind, sham-controlled, randomized trial evaluating venous angioplasty to address extracranial venous anomalies characterized as CCSVI in patients with MS, we found that the procedure was reasonably safe. However, it failed to provide any sustained improvement in venous outflow as measured through duplex or clinical and MRI outcomes. To the contrary, more sizeable change in venous outflow was associated with increased disease activity primarily noted on MRI. This study was a limited pilot trial not powered to detect possible safety concerns observable only with a larger population and longer follow-up; however, the results caution against widespread adoption of venous angioplasty in the management of patients with MS outside of rigorous clinical trials. It also provides validation for conduct of shamcontrolled, double-blind trials in the evaluation of novel interventions in complex diseases.

\section{AUTHOR CONTRIBUTIONS}

Conception and design: Dr. Siddiqui, Dr. Zivadinov, Dr. WeinstockGuttman, Dr. Karmon. Acquisition of data: All authors. Analysis and interpretation of data: All authors. Drafting the manuscript: Dr. Siddiqui, Dr. Zivadinov. Critically revising the manuscript: All authors. Final approval of the manuscript: All authors. Statistical analysis: Dr. Yu. Administrative, technical, or material support: Dr. Siddiqui, Dr. Zivadinov, Dr. Karmon. Study supervision: Dr. Siddiqui, Dr. Zivadinov, Dr. Weinstock-Guttman, Dr. Karmon.

\section{ACKNOWLEDGMENT}

The authors thank all study subjects for their participation; Data Safety Monitoring Committee members Robert Sawyer Jr., MD, and Robert J. Plunkett, MD, for their assistance during the trial; Paul H. Dressel, BFA, for assistance with preparation of the illustrations; and Debra J. Zimmer for editorial support.

\section{STUDY FUNDING}

Kaleida Health (New York) in the form of provision of diagnostic and interventional services at no cost for the study; Direct MS Foundation (Canada), Volcano Corp (California), Covidien/ev3 Corp (California), and Jacquemin Family Foundation (Virginia) in the form of unrestricted educational grants or donations to Kaleida Health or to the University at Buffalo, State University of New York. This was an investigator-initiated study and there was no involvement from any of the sponsoring organizations in the design, collection, analysis, interpretation of data, writing of the report, or submission for publication.

\section{DISCLOSURE}

Dr. Siddiqui: research grants: National Institutes of Health (not related to present study) and University at Buffalo; financial interests: Blockade Medical, Hotspur, Intratech Medical, Lazarus Effect, StimSox, Valor Medical; consultant: Blockade Medical, Codman \& Shurtleff, Inc., Concentric Medical, ev3/Covidien Vascular Therapies, GuidePoint Global Consulting, Lazarus Effect, MicroVention, Penumbra, Stryker, Pulsar Vascular; National Steering Committee: 3D Separator Trial (Penumbra, Inc.), SWIFT PRIME Trial (Covidien), FRED Trial (MicroVention); speakers' bureau: Codman \& Shurtleff, Inc.; advisory board: Codman \& Shurtleff, Inc., Covidien Neurovascular; honoraria: Abbott Vascular and Codman \& Shurtleff, Inc. for training other physicians in carotid stenting and endovascular stenting for aneurysms, and Penumbra, Inc. Dr. Zivadinov: financial support for research activities from Biogen Idec, Teva Pharmaceutical and Teva Neuroscience, EMD Serono, GenzymeSanofi, Novartis, Greatbatch, Bracco, and Questcor; personal compensation from Teva Pharmaceutical, Biogen Idec, Novartis, Genzyme-Sanofi, EMD Serono, Bayer, Novartis, and General Electric for speaking and consultant services. Dr. Benedict: speaker's bureaus for EMD Serono; consultant for Biogen Idec, Novartis, and Bayer; grant/research support from Biogen Idec, Accorda, and Shire. Dr. Karmon, Dr. Yu, M. Hartney, K. Marr, Dr. Valnarov, and C. Kennedy report no disclosures relevant to the manuscript. Dr. Ramanathan: research funding or consulting fees from EMD Serono, Biogen Idec, Allergan, Netezza, Pfizer, Novartis, the National Multiple Sclerosis Society, the Department of Defense, Jog for the Jake Foundation, the National Institutes of Health, and National Science Foundation; compensation for serving as an Editor from the American Association of Pharmaceutical Scientists. Dr. Ramasamy and Dr. Dolic report no disclosures relevant to the manuscript. Dr. Hojnacki: speaker honoraria and consultant fees from Biogen Idec, Teva Pharmaceutical Industries Ltd., EMD Serono, Pfizer Inc., and Genzyme. Dr. Carl reports no disclosures relevant to the manuscript. Dr. Levy: shareholder/ownership interests: Intratech Medical Ltd., Mynx/Access Closure, Blockade Medical LLC. Principal investigator: Covidien US SWIFT PRIME Trials. Other financial support: Abbott for carotid training for physicians. L. Hopkins: grant/research support from Toshiba; consultant to Abbott, Boston Scientific, Cordis, Micrus, and Silk Road; financial interests in AccessClosure, Augmenix, Boston Scientific, Claret Medical, Endomation, Micrus, and Valor Medical; board/trustee/officer position with Access Closure and Claret Medical; Abbott Vascular's speakers' bureau; honoraria from Bard, Boston Scientific, Cleveland Clinic, Complete Conference Management, Cordis, Memorial Health Care System, and the SCAI. Dr. Weinstock-Guttman: speaker's bureaus and consultant for Biogen Idec, Teva Neurosciences, EMD Serono, Pfizer, Novartis, Genzyme, and Acorda; grant/research support from the agencies listed above as well as ITN, Questcor, and Shire. Go to Neurology. org for full disclosures.

Received September 30, 2013. Accepted in final form March 17, 2014.

\section{REFERENCES}

1. Zamboni P, Galeotti R, Menegatti E, et al. Chronic cerebrospinal venous insufficiency in patients with multiple sclerosis. J Neurol Neurosurg Psychiatry 2009;80:392-399.

2. Baracchini C, Perini P, Calabrese M, Causin F, Rinaldi F, Gallo P. No evidence of chronic cerebrospinal venous insufficiency at multiple sclerosis onset. Ann Neurol 2011;69:90-99.

3. Baracchini C, Perini P, Causin F, Calabrese M, Rinaldi F, Gallo P. Progressive multiple sclerosis is not associated with chronic cerebrospinal venous insufficiency. Neurology 2011; 77:844-850.

4. Barreto AD, Brod SA, Bui TT, et al. Chronic cerebrospinal venous insufficiency: case-control neurosonography results. Ann Neurol 2013;73:721-728.

5. Doepp F, Paul F, Valdueza JM, Schmierer K, Schreiber SJ. No cerebrocervical venous congestion in patients with multiple sclerosis. Ann Neurol 2010;68:173-183.

6. Tsivgoulis G, Mantatzis M, Bogiatzi C, et al. Extracranial venous hemodynamics in multiple sclerosis: a case-control study. Neurology 2011;77:1241-1245.

7. Wattjes MP, van Oosten BW, de Graaf WL, et al. No association of abnormal cranial venous drainage with multiple sclerosis: a magnetic resonance venography and flowquantification study. J Neurol Neurosurg Psychiatry 2011; 82:429-435.

8. Zamboni P, Galeotti R, Menegatti E, et al. A prospective open-label study of endovascular treatment of chronic 
cerebrospinal venous insufficiency. J Vasc Surg 2009;50: $1348-1358$

9. Alroughani R, Lamdhade S, Thussu A. Endovascular treatment of chronic cerebrospinal venous insufficiency in multiple sclerosis: a retrospective study. Int J Neurosci 2013; 123:324-328.

10. Ghezzi A, Annovazzi P, Cocco E, et al. Endovascular treatment of CCSVI in patients with multiple sclerosis: clinical outcome of 462 cases. Neurol Sci 2013;34:1633-1637.

11. Hubbard D, Ponec D, Gooding J, Saxon R, Sauder H, Haacke M. Clinical improvement after extracranial venoplasty in multiple sclerosis. J Vasc Interv Radiol 2012;23: 1302-1308.

12. Kipshidze N, Rukhadze I, Archvadze A, Kipiani V, Lapiashvili E, Kaloiani V. Endovascular treatment of patients with chronic cerebrospinal venous insufficiency and multiple sclerosis. Georgian Med News 2011:29-34.

13. Ludyga T, Kazibudzki M, Simka M, et al. Endovascular treatment for chronic cerebrospinal venous insufficiency: is the procedure safe? Phlebology 2010;25:286-295.

14. Mandato KD, Hegener PF, Siskin GP, et al. Safety of endovascular treatment of chronic cerebrospinal venous insufficiency: a report of 240 patients with multiple sclerosis. J Vasc Interv Radiol 2012;23:55-59.

15. Petrov I, Grozdinski L, Kaninski G, Iliev N, Iloska M, Radev A. Safety profile of endovascular treatment for chronic cerebrospinal venous insufficiency in patients with multiple sclerosis. J Endovasc Ther 2011;18:314-323.

16. Radak D, Kolar J, Sagic D, et al. Percutaneous angioplasty of internal jugular and azygous veins in patients with chronic cerebrospinal venous insufficiency and multiple sclerosis: early and mid-term results. Phlebology Epub 2013 May 3.

17. Salvi F, Bartolomei I, Buccellato E, Galeotti R, Zamboni P. Venous angioplasty in multiple sclerosis: neurological outcome at two years in a cohort of relapsingremitting patients. Funct Neurol 2012;27:55-59.

18. Zamboni P, Galeotti R, Weinstock-Guttman B, Kennedy C, Salvi F, Zivadinov R. Venous angioplasty in patients with multiple sclerosis: results of a pilot study. Eur J Vasc Endovasc Surg 2012;43:116-122.

19. Ghezzi A, Annovazzi P, Amato M, et al. Adverse events after endovascular treatment of chronic cerebrospinal venous insufficiency (CCSVI) in patients with multiple sclerosis. Mult Scler 2013;19:961-963.

20. Kurtzke JF. Rating neurologic impairment in multiple sclerosis: an expanded disability status scale (EDSS). Neurology 1983;33:1444-1452.

21. Polman CH, Reingold SC, Banwell B, et al. Diagnostic criteria for multiple sclerosis: 2010 revisions to the McDonald criteria. Ann Neurol 2011;69:292-302.

22. Zivadinov R, Marr K, Cutter G, et al. Prevalence, sensitivity, and specificity of chronic cerebrospinal venous insufficiency in MS. Neurology 2011;77:138-144.

23. Lublin FD, Reingold SC. Defining the clinical course of multiple sclerosis: results of an international survey: National Multiple Sclerosis Society (USA) Advisory
Committee on Clinical Trials of New Agents in Multiple Sclerosis. Neurology 1996;46:907-911.

24. Karmon Y, Zivadinov R, Weinstock-Guttman B, et al. Comparison of intravascular ultrasound with conventional venography for detection of extracranial venous abnormalities indicative of chronic cerebrospinal venous insufficiency. J Vasc Interv Radiol 2013;24:1487-1498.

25. Noguchi K, Konietschke F, Gel Y, Brunner E. nparLD: an $\mathrm{R}$ software package for the nonparametric analysis of longitudinal data in factorial experiments. J Stat Softw 2012; 50:1-23.

26. Brod SA, Lindsey JW, Vriesendorp FS, et al. Ingested IFN-alpha: results of a pilot study in relapsing-remitting MS. Neurology 2001;57:845-852.

27. Frohman EM, Cutter G, Remington G, et al. A randomized, blinded, parallel-group, pilot trial of mycophenolate mofetil (CellCept) compared with interferon beta-1a (Avonex) in patients with relapsing-remitting multiple sclerosis. Ther Adv Neurol Disord 2010;3:15-28.

28. Tubridy N, Ader HJ, Barkhof F, Thompson AJ, Miller DH. Exploratory treatment trials in multiple sclerosis using MRI: sample size calculations for relapsingremitting and secondary progressive subgroups using placebo controlled parallel groups. J Neurol Neurosurg Psychiatry 1998;64:50-55.

29. Visser LH, Beekman R, Tijssen CC, et al. A randomized, double-blind, placebo-controlled pilot study of i.v. immune globulins in combination with i.v. methylprednisolone in the treatment of relapses in patients with MS. Mult Scler 2004;10:89-91.

30. Zivadinov R, Ramanathan M, Dolic K, et al. Chronic cerebrospinal venous insufficiency in multiple sclerosis: diagnostic, pathogenetic, clinical and treatment perspectives. Expert Rev Neurother 2011;11:1277-1294.

31. Zivadinov R, Weinstock-Guttman B. Role of venoplasty for treatment of multiple sclerosis: value of open-label studies and surrogate treatment outcomes. J Vasc Interv Radiol 2012;23:1308-1310.

32. Dolic K, Marr K, Valnarov V, et al. Sensitivity and specificity for screening of chronic cerebrospinal venous insufficiency using a multimodal non-invasive imaging approach in patients with multiple sclerosis. Funct Neurol 2011;26:205-214.

33. Dolic K, Marr K, Valnarov V, et al. Intra- and extraluminal structural and functional venous anomalies in multiple sclerosis, as evidenced by 2 noninvasive imaging techniques. AJNR Am J Neuroradiol 2012;33:16-23.

34. Traboulsee AL, Knox KB, Machan L, et al. Prevalence of extracranial venous narrowing on catheter venography in people with multiple sclerosis, their siblings, and unrelated healthy controls: a blinded, case-control study. Lancet 2014;383:138-145.

35. Denislic M, Milosevic Z, Zorc M, Ravnik IZ, Mendiz O. Disability caused by multiple sclerosis is associated with the number of extra cranial venous stenoses: possible improvement by venous angioplasty: results of a prospective study. Phlebology 2013;28:353-360. 